\title{
Development and Evaluation of Adjoint Approach for Aeroelastic Wing Optimization
}

\author{
Mohammad Abu-Zurayk, Joël Brezillon
}

\begin{abstract}
In the context of gradient-based optimization techniques for multidisciplinary problems an efficient approach was sought to evaluate the gradient of the cost function with respect to the design variables; also called the sensitivities. The traditional approach to calculate the sensitivities, the finite differences, can become prohibitively expensive in high-fidelity optimizations. For this reason an existing adjoint approach was suggested to be further developed in order to suit coupled aero-structural systems. Then the developed approach was evaluated and tested. The results showed that the approach can provide accurate sensitivities in a very efficient way.
\end{abstract}

\section{Introduction}

Computing the sensitivities for gradient-based multidisciplinary optimization techniques with the traditional finite differences approach can be extremely expensive. If a coupled aero-structural system is considered for example, the sensitivities require two converged coupled computations for each design parameter with the central finite differences approach, which means that such technique can become prohibitively expensive for high-fidelity problems with large number of design parameters, as the computational power needed is linearly dependent on the number of design parameters.

To get rid of this expensive dependency, the discrete adjoint approach is investigated here. In this approach, a Lagrange formulation is defined and used in such a way that eliminates this dependency, where only one converged coupled computation and one adjoint computation are needed to compute the sensitivities. The flow

Mohammad Abu-Zurayk

DLR, Lilienthalplatz 7, 38108 Braunschweig, e-mail: mohammad.abu-zurayk@dlr.de

Joël Brezillon

DLR, Lilienthalplatz 7, 38108 Braunschweig e-mail: joel.brezillon@ dlr.de 
adjoint technique has been successfully developed in the DLR CFD code; TAU [2], then tested for NS equations in 3D configurations [3], and has proven to be very efficient and cheap in comparison to finite differences. For this reason, this approach was suggested to be further developed for coupled aero-structural systems in the frame of the internal DLR-funded project; MDOrmec.

\section{Definition and formulation of the problem}

The coupled aero-structure adjoint formulation takes the effect of the structure displacements into account while solving for the gradients of a defined cost function (I) with respect to some design variables (D). The following describes the suggested formulation of the adjoint equations for a coupled aero-structure system.

The system to be solved can be defined as follows. The state variables of the coupled system is the vector $\mathrm{W}=[\mathrm{w} \mathrm{u}]$; where $(\mathrm{w})$ represents the flow variables and (u) represents the structure displacements. The design variables of this system are defined by $\mathrm{D}=[\mathrm{A} \mathrm{T}]$; where $(\mathrm{A})$ and $(\mathrm{T})$ are the shape design variables and the thickness of the structure elements, respectively. The cost function to be optimized (I) can be defined as a function of both the state and the design variables; I=I(W,D). Similarly the aerodynamic residual $(\mathrm{Ra})$ and the structure residual (Rs) are defined; $\mathrm{Ra}=\mathrm{Ra}(\mathrm{W}, \mathrm{A})$ and $\mathrm{Rs}=\mathrm{Rs}(\mathrm{W}, \mathrm{T})$. Here the aerodynamic residuals are described by the flow equations (Euler or Navier-Stokes) and the structure equation is described by

$$
R s=K u-f=0,
$$

where (K) is the Stiffness Matrix, (u) is the structural displacement and (f) represents the forces on the structure nodes.

The gradient of the cost function with respect to the design variables can be written in the vector format as:

$$
\frac{d I}{d D}=\frac{\partial I}{\partial D}+\left[\begin{array}{ll}
\frac{\partial I}{\partial w} & \frac{\partial I}{\partial u}
\end{array}\right]\left[\begin{array}{l}
\frac{d w}{d D} \\
\frac{d u}{d D}
\end{array}\right] .
$$

On the other hand the gradients of the aerodynamic and the structure residuals with respect to the vector of design variables have the same format as that of the cost function. To formulate the coupled adjoint equation, a Lagrangian $(\mathrm{L})$ is defined as:

$$
L=I+\Psi R,
$$

where the Lagrange multiplier

$$
\Psi=[\psi \phi]
$$

contains both the aerodynamic $\psi$ and structure $\phi$ multipliers respectively, and the vector $\mathrm{R}=[\mathrm{Ra} \mathrm{Rs}]$ represents the residuals vector that contains both aerodynamic and structure residuals, respectively. After defining the Lagrangian, its gradient with respect to the design variables is sought. Having the residual vector $R=0$, the gra- 
dient of the Lagrangian is equal to that of the cost function. The gradient of the Lagrangian is:

$$
\begin{aligned}
\frac{d L}{d D}=\frac{d I}{d D}= & \left(\left[\begin{array}{ll}
\frac{\partial I}{\partial A} & \frac{\partial I}{\partial T}
\end{array}\right]+[\psi \phi]\left[\begin{array}{ll}
\frac{\partial R a}{\partial A} & \frac{\partial R a}{\partial T} \\
\frac{\partial R s}{\partial A} & \frac{\partial R s}{\partial T}
\end{array}\right]\right)\left(\left[\begin{array}{l}
\frac{d A}{d D} \\
\frac{d T}{d D}
\end{array}\right]\right) \\
& +\left(\left[\begin{array}{ll}
\frac{\partial I}{\partial w} & \frac{\partial I}{\partial u}
\end{array}\right]+[\psi \phi]\left[\begin{array}{ll}
\frac{\partial R a}{\partial w} & \frac{\partial R a}{\partial u} \\
\frac{\partial R s}{\partial w} & \frac{\partial R s}{\partial u}
\end{array}\right]\right)\left(\left[\begin{array}{l}
\frac{d w}{d D} \\
\frac{d u}{d D}
\end{array}\right]\right) .
\end{aligned}
$$

The expensive terms in this equation are the ones that relate the flow and the structure state variables to the design variables, namely $(\mathrm{dw} / \mathrm{dD})$ and $(\mathrm{du} / \mathrm{dD})$. They are expensive because they force the designer to perform one flow or structure computation for each design variable, which for high fidelity design or optimization can be extremely expensive. To get rid of these terms, the second part of the RHS in this equation is set to zero by finding the suitable vector $\Psi$ that fulfils this condition. This vector is not predefined, which means that it can be chosen to satisfy a described condition, and the condition in this case is to have this part of the equation equal to zero. This results in:

$$
\left[\begin{array}{ll}
\frac{\partial I}{\partial w} & \frac{\partial I}{\partial u}
\end{array}\right]^{T}+\left(\left[\begin{array}{ll}
\psi & \phi
\end{array}\right]\left[\begin{array}{ll}
\frac{\partial R a}{\partial w} & \frac{\partial R a}{\partial u} \\
\frac{\partial R s}{\partial w} & \frac{\partial R s}{\partial u}
\end{array}\right]\right)^{T}=0
$$

Equation (6) is called the coupled adjoint equation and is independent of the number of design variables. As soon as it is solved, the Lagrange multipliers vector $\Psi$ is found and used to solve the first part of equation (5)to get the gradients of the cost function w.r.t the design variables.

$$
\frac{d I}{d D}=\left(\left[\begin{array}{ll}
\frac{\partial I}{\partial A} & \frac{\partial I}{\partial T}
\end{array}\right]+[\psi \phi]\left[\begin{array}{ll}
\frac{\partial R a}{\partial A} & \frac{\partial R a}{\partial T} \\
\frac{\partial R S}{\partial A} & \frac{\partial R S}{\partial T}
\end{array}\right]\right)\left(\left[\begin{array}{l}
\frac{d A}{d D} \\
\frac{d T}{d D}
\end{array}\right]\right)
$$

This means that the solution of only one aero-structure coupling, one coupled adjoint system of equations and one gradient equation will provide the sensitivities.

To test the coupled adjoint approach, it was suggested, as a start, to consider a cost function that is exclusively related to aerodynamic terms, like drag, lift or a combination of both. The effect of the structure displacements will still be taken into account, and the resulting sensitivities will include information about the static aeroelasticity of the problem. As a consequence, the terms that depend on the structure variables (T), will be pushed to zero, and the gradient equation (7) will shrink to:

$$
\frac{d I}{d D}=\frac{\partial I}{\partial D}+\psi \frac{\partial R a}{\partial D}+\phi \frac{\partial R s}{\partial D}
$$




\section{Implementation and resolution of the coupled adjoint system}

After defining the problem in a simplified manner, the next step was to detect the terms of the coupled adjoint system and to check which of these terms is efficiently obtainable and which needs to be derived in order to avoid costly computations of these terms.

In the system of equations (6), the terms related to pure aerodynamic adjoint are already derived, namely $\left(\frac{\partial I}{\partial w}\right)$ and $\left(\frac{\partial R a}{\partial w}\right)$, and the rest should be derived for the purpose of this work. The first term to be derived in this system of equations is the term $\left(\frac{\partial I}{\partial u}\right)$ which represents the sensitivity of the cost function with respect to the displacement in the structural mesh. Another term that depends on this displacement is the term $\left(\frac{\partial R a}{\partial u}\right)$ which represents the sensitivity of the aerodynamic residuals with respect to the displacement in the structure mesh. If the displacement in the structural mesh changes, the aerodynamic mesh will also change and this affects both the aerodynamic cost function and the aerodynamic residual. Those two terms can be rewritten as:

$$
\frac{\partial I}{\partial u}=\frac{\partial I}{\partial X a} \frac{\partial X a}{\partial u}, \frac{\partial R a}{\partial u}=\frac{\partial R a}{\partial X a} \frac{\partial X a}{\partial u},
$$

respectively, where Xa represents the aerodynamic volume mesh.

By knowing that the terms $\left(\frac{\partial I}{\partial X a}\right)$ and $\left(\frac{\partial R a}{\partial X a}\right)$ are already derived in the flow solver TAU [6], it becomes clear that only one term $\left(\frac{\partial X a}{\partial u}\right)$ should be derived here, instead of deriving both $\left(\frac{\partial I}{\partial u}\right)$ and $\left(\frac{\partial R a}{\partial u}\right)$. This term represents the sensitivity of the movement in the aerodynamic mesh with respect to that in the structural mesh, which means that this term depends directly on the interpolation between meshes that is used during the fluid-structure coupling. In this case the mesh interpolation tool used for coupling is the Radial Basis Function (RBF) tool developed in TAU.

The term $\left(\frac{\partial X a}{\partial u}\right)$ was found by differentiating the RBF interpolation tool as explained in [5], and the results were evaluated against values computed by the costly finite differences on a 3D LANN wing test case. Figure (1a) shows excellent matching in the results.

The second term to be derived is $\left(\frac{\partial R s}{\partial w}\right)$ which represents the sensitivity of the structural residuals with respect to the change in the flow state variables. While it is possible in the DLR-TAU solver to exchange between the conservative and the primitive variables, this term shall be derived with respect to the primitive variables, as this makes the derivation much easier.

According to equation (1), only the force term (F) is of interest here,

$$
\frac{\partial R s}{\partial w}=\frac{-\partial F}{\partial w}=-\frac{\partial F}{\partial p},
$$

where $\mathrm{p}$ is the pressure; the only primitive variable that directly affects the force. This term is found by differentiating the procedure that interpolates the pressure from the CFD mesh and transfers it into forces over the CSM mesh. In this work, a linear interpolation tool is used. 


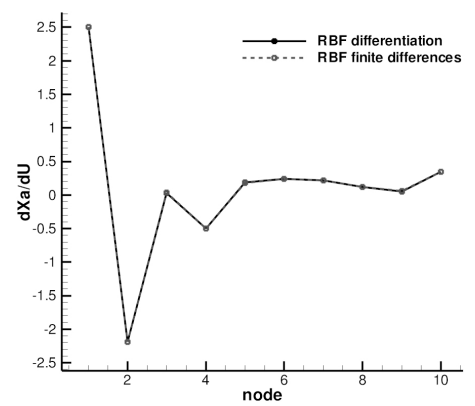

(a) evaluation of the differentiation of the Mesh interpolation tool (term $\frac{\partial X a}{\partial u}$ ) for LANN wing

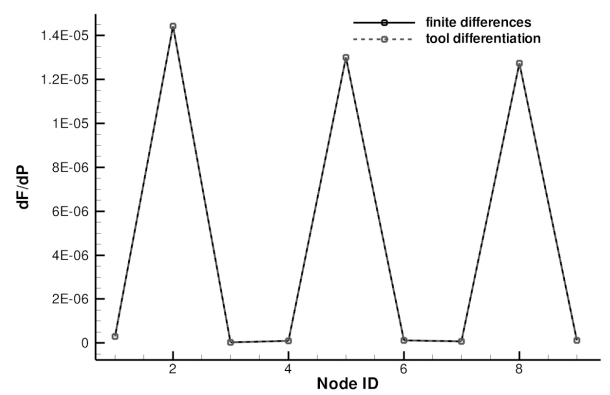

(b) evaluation of the differentiation of the pressure interpolation tool (term $\frac{\partial R s}{\partial w}$ ) for LANN wing

Fig. 1: Evaluation of the derived terms

To test and evaluate this term, it was compared to the results of finite differences. The test was done on some nodes of the LANN wing in different directions and the result of this comparison can be seen in figure $1 b$.

The last term to be found to solve the coupled adjoint system of equations is $\left(\frac{\partial R s}{\partial u}\right)$ which represents the sensitivity of the structural residual with respect to the structural displacement. This term is equal to the stiffness matrix $(\mathrm{K})$ that is present in the structural residual equation 1 .

After deriving all the required terms in the described system of equations, a way to solve the coupled adjoint system of equations is investigated. Such system of equations is usually solved through the lagged iterative method [1]. After arranging the system of equations it will look as the following:

$$
\begin{gathered}
\frac{\partial R a^{T}}{\partial w} \psi^{T n}=-\frac{\partial I^{T}}{\partial w}-\frac{\partial R s^{T}}{\partial w} \phi^{T n-1} \\
\frac{\partial R a^{T}}{\partial w} \psi^{T n}=-\frac{\partial I^{T}}{\partial w}-\frac{\partial R s^{T}}{\partial w} \phi^{T n}
\end{gathered}
$$

The lagged iterative method suggests initializing the process for $\phi^{n-1}=0$, then solving the first equation for $\psi^{T n}$, after that solving the second equation for $\phi^{n}$ and keep iteratively solving those two equations until a convergence criteria is reached. The system can now be solved and the adjoint fields can be computed and inserted in the gradient equation.

\section{Implementation of the gradient equation}

Once the coupled adjoint field is computed, the last step consists in computing the gradient by solving the equation 8 which can be rewritten as: 


$$
\frac{d I}{d D}=\frac{\partial I}{\partial X a} \frac{\partial X a}{\partial D}+\psi \frac{\partial R a}{\partial X a} \frac{\partial X a}{\partial D}+\phi \frac{\partial R s}{\partial D}
$$

The missing terms are $\left(\frac{\partial X a}{\partial D}\right)$ and $\left(\frac{\partial R s}{\partial D}\right)$. The first term describes how the aerodynamic mesh at the flight shape changes by moving the design variables. The second term represents the sensitivity of the structure residuals with respect to the movement in the design variables, in this term, the change in the stiffness matrix due to the movement of the design variables is neglected, and only the change in the structural forces is taken into account. Both terms are computed through finite differences because they can be obtained cheaply in this way.

\section{Results}

To validate the developed technique, the previously mentioned test case; 3D LANN wing, was used. The wing has around 10000 grid nodes on the structure side and around 30000 grid nodes on the CFD side. The solver used on the structure side is ANSYS and DLR-TAU is used to solve the CFD Euler equations. The validation included the gradients of drag and lift, and was performed at Mach number of 0.84 and an angle of attack of 0.6 degree. The wing is described using 60 design variables that are defined by the free form deformation technique [7]. 36 design variables were used to evaluate the gradients, Figure 2 presents the evaluation for drag and lift respectively.

Even though only aerodynamic design variables were employed, the effect of the static aeroelasticity was taken into account while computing the gradients. The figure shows a very good matching of gradients. The time needed to get the gradients with the central finite differences approach is around 72 hours, where only 5 hours were needed to get the gradients using the coupled adjoint formulation; 2.5 hours for each cost function.

To bring the coupled adjoint approach into application, a drag reduction optimization was performed under the constraint of constant lift. The previous test case was taken for this optimization, where the python-based optimization environment used; Pyranha, [4] employed a conjugate gradient optimization algorithm. Figure 3 shows the convergence of the cost function throughout the optimization.

Figure 4 shows the airfoil profile and the pressure coefficient at $\eta=0.5$ of the wing for the optimization, where the dashed lines represents the initial states and the solid lines represent the optimized states. Figure 3 shows large reduction in the drag at constant lift. While the optimization resulted in decrease in the airfoil thickness, another optimization was performed at constant airfoil thickness and showed less but satisfactory reduction in the drag at constant lift. 


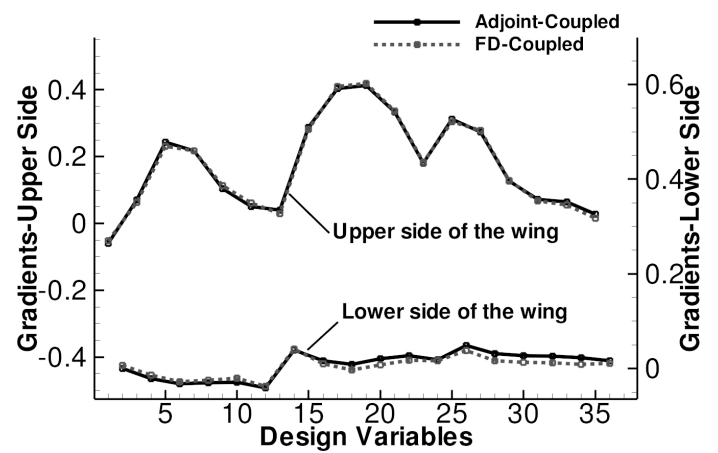

(a) Drag Gradients

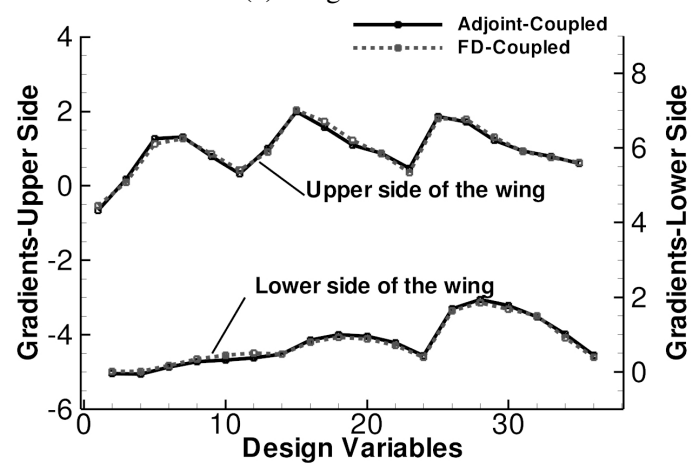

(b) Lift Gradients

Fig. 2: Gradients evaluation

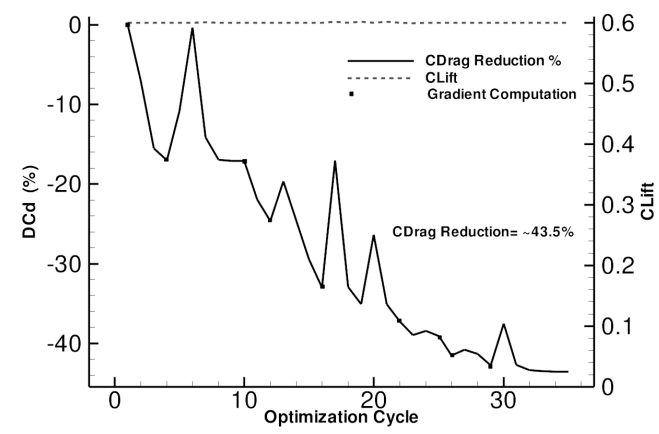

Fig. 3: Optimization convergence 


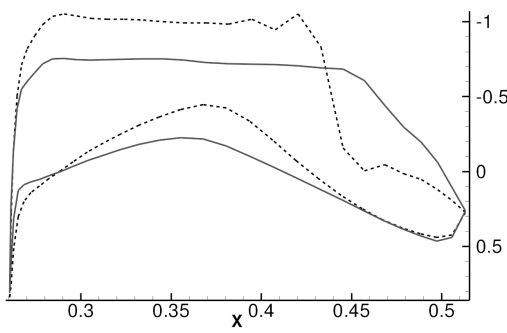

(a) Cp distributions at $\eta=0.5$

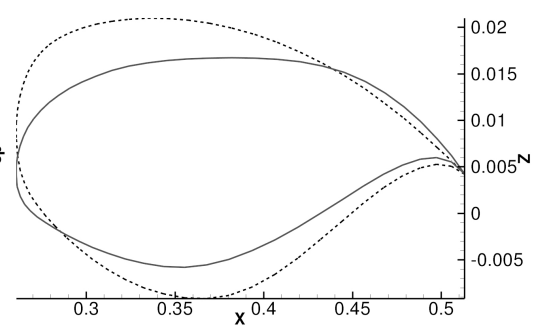

(b) airfoils at $\eta=0.5$

Fig. 4: Initial and Optimized states at $\eta=0.5$

\section{Conclusions}

In this study, the adjoint approach for a coupled aero-structural system was successfully derived and implemented. The coupled system was solved using TAU on the CFD side and ANSYS on the CSM side. The advantages of such approach are to compute accurate cost functions gradients and to considerably reduce the time needed to compute them compared with the traditional approach. After evaluating the gradients obtained by the adjoint approach, two optimizations were performed, once reducing the drag at constant lift, and the other at constant lift and thickness. Both optimizations showed large drag decrease.

This work will be further developed in order to take structural cost functions into account, and to include the Navier-Stokes equations on the CFD side.

\section{References}

1. Martins, J. Alonso, J. Reuther, J. A Coupled-Adjoint Sensitivity Analysis Method for HighFidelity Aero-Structural Design. Optimization and Engineering,6, 33-62 (2005)

2. Dwight, R. Efficiency Improvements of RANS-Based Analysis and Optimization using Implicit and Adjoint Methods on Unstructured Grids, DLR Forschungsbericht. (2006)

3. Brezillon, J and Dwight, R. Aerodynamic Shape Optimization Using the Discrete Adjoint of the Navier-Stokes Equations: Application towards Complex 3D Configurations. KATnet paper. (2009)

4. Brezillon, J. Abu-Zurayk, M. Aerodynamic Inverse Design Framework using Discrete Adjoint Method, 17 DGLR-Fach-Symposium der STAB, 9-10 November 2010, Berlin

5. Jakobsson, S. and Amoignon, O. Mesh Deformationusing Radial Basis Function for Gradientbased Aerodynamic Shape Optimization. FOI-R-1784-SE (2005)

6. Widhalm, M. Brezillon, J. Ilic, C. Leicht, T. Investigation on Adjoint Based Gradient Computations for Realistic 3d Aero-Optimization, 13th AIAA/ISSMO Multidisciplinary Analysis Optimization Conference 13 - 15 Sep 2010, Fort Worth, Texas

7. Ronzheimer, A. Shape Parametrization Using Freeform Deformation. In: MEGAFLOW Numerical Flow Simulation for Aircraft Design, 89, pp. 211-222. Springer Verlag. Closing Presentation DLR Project MEGAFLOW, Braunschweig (de), 10.-11.12.2002. ISBN 3-54024383-6 (2005). 